\title{
An Unusual Presentation of Disseminated Cryptococcosis in a Middle Aged Female
}

\author{
Dipankar Pal ${ }^{1 *}$, Avijit Das ${ }^{1}$, Shekhar Pal ${ }^{1}$, Soumyadip Chatterji ${ }^{1}$, Manab Kumar Ghosh ${ }^{1}$, Arindam Naskar ${ }^{1}$, Sudeshna Mallik ${ }^{1}$ and Abhiram Chakrabarty ${ }^{2}$ \\ ${ }^{1}$ Department of Tropical Medicine, School of Tropical Medicine, Kolkata, India \\ ${ }^{2}$ Department of Radiology, Calcutta National Medical College, Kolkata
}

"Corresponding author: Dipankar Pal, RMO cum Clinical Tutor, Department of Tropical Medicine, School of Tropical Medicine, Kolkata, India, Phone: +91-9432113713; E-mail: dipankarpal.2009@gmail.com

Received date: March 5, 2015, Accepted date: April 14, 2015, Published date: April 16, 2015

Copyright: $\odot 2015$ Dipankar P et al. This is an open-access article distributed under the terms of the Creative Commons Attribution License, which permits unrestricted use, distribution, and reproduction in any medium, provided the original author and source are credited.

\begin{abstract}
Cryptococcosis is a common opportunistic pathogen in the setting of advanced human immunodeficiency virus (HIV) infection especially with CD4 count less than 50 cells/micro liter and in other immunodeficient conditions such as hematologic malignancies, transplant recipients or in persons on prolonged glucocorticoid and/or immunosuppressant therapy. Disseminated cryptococcosis is uncommon in immunocompetent person. We detected such a case at our institute presented initially with pulmonary mass lesion and later evolving into chronic non-healing skin ulcer and asymptomatic brain involvement without having any evidence of overt immunosuppression. However the patient developed gall-bladder carcinoma about one and half years after her chest complaints. She underwent surgical resection but neither received radiotherapy nor chemotherapy post-surgery. She was treated successfully with anti-fungal combination therapy as per Infectious Diseases Society of America (IDSA) guideline.
\end{abstract}

Keywords: Disseminated Cryptococcosis; Lung mass lesion; Nonhealing skin ulcer; Asymptomatic brain involvement; Atypical neuroimaging

\section{Introduction}

Cryptococcosis is caused by an encapsulated fungus called Cryptococcus neoformans. It has a worldwide distribution and mainly acquired from environment by inhalation of aerosolised infectious particles. It is an opportunistic yeast found frequently in soil contaminated with pigeon and other bird droppings. It can affect any tissue or organ, but majority of cases that come to clinical attention involves the central nervous system (CNS) and/or lungs [1]. The clinical features of cryptococcosis differ between HIV-infected and non-infected persons. Meningoencephalitis is predominant in HIVpositive patients, whereas HIV-negative patients generally present with pulmonary or central nervous system space occupying lesion [2].

\section{Case Report}

A 44 years old non-diabetic female from rural West Bengal was suffering from low grade intermittent fever, dry cough, fatigue and weight loss for one and half year. She consulted her physician and underwent testing of blood and sputum but without any clue. Her chest-X ray revealed homogenous opacity with a smooth margin in left upper and mid zone. Few weeks later, she started to have episodes of haemoptysis along with pain over left upper chest. Contrast enhanced CT (CECT) scan thorax was done after one month (Figure 1) along with a guided FNAC from left lung lesion which revealed granulomatous pathology without evidence of any acid fast bacilli (AFB).

She was put on anti-tubercular drugs (ATD) empirically (rifampicin, INH, pyrazinamide and ethambutol). But after two months she started to show signs and symptoms of drug-induced hepatitis.

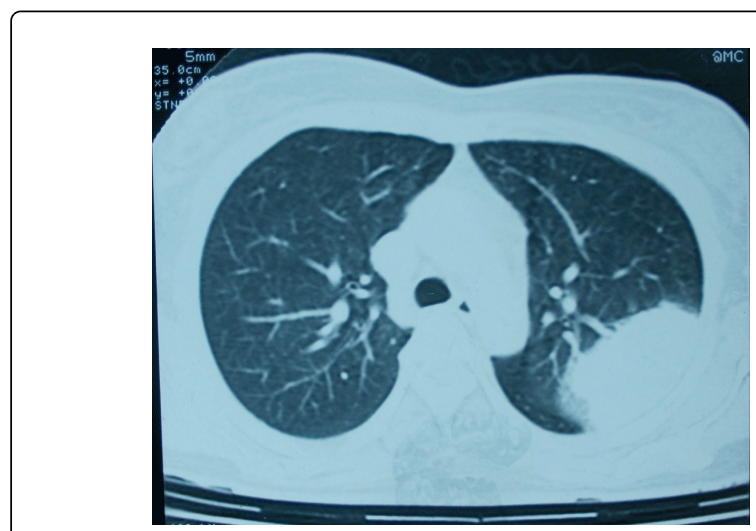

Figure 1: CECT thorax at presentation.

Subsequently her ATD was changed to hepatic friendly regimen (ethambutol, streptomycin and levofloxacin) for four months. However there was no improvement of her respiratory symptoms and she was being treated symptomatically by her care givers for about one year.

Thereafter she felt some dull aching pain in right upper abdomen which on evaluation was found to be due to a gall bladder (GB) mass involving cystic duct and neck of GB. There was no previous history of pain abdomen, jaundice, nausea, vomiting. Radical cholecystectomy with extrahepatic bile duct excision and hepatico - jejunostomy was done. Histopathology of the resected specimen revealed adenocarcinoma of GB. No post-surgical chemo or radiotherapy was advised by the oncologist. 
Citation: $\quad$ Dipankar P, Avijit D, Shekhar P, Soumyadip C, Manab KG, et al. (2015) An Unusual Presentation of Disseminated Cryptococcosis in a Middle Aged Female. Trop Med Surg 3: 183. doi:10.4172/2329-9088.1000183

Page 2 of 4

Few weeks after surgery, she noticed a small nodule over anterior aspect of right arm just above the elbow joint. The nodule was skin coloured, mobile, painless and gradually increased in size over next 6 months into a fungating lesion of about $6 \mathrm{~cm}$ in long axis (Figure 2a).

It did not respond to local and systemic antibiotics. Her chest symptoms also continued for that entire period. Six months later, biopsy from the skin lesion showed thick walled budding yeasts. Thereafter she was referred to our institute.
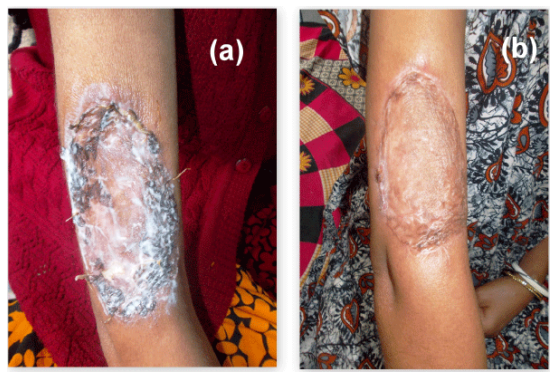

Figure 2: Showing skin ulcer before (a) and after (b) treatment.

Chest X-Ray done on admission to our institute (Figure 3a) showed a moderate to large homogenous opacity involving left upper and mid zone.

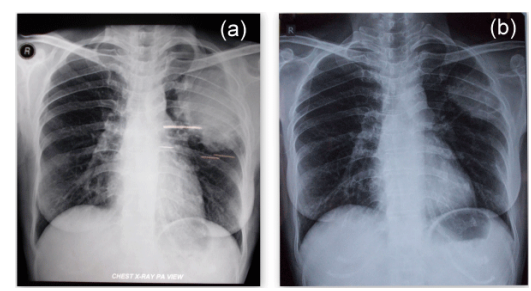

Figure 3: Showing chest $\mathrm{x}$-ray before (a) and after (b) treatment.

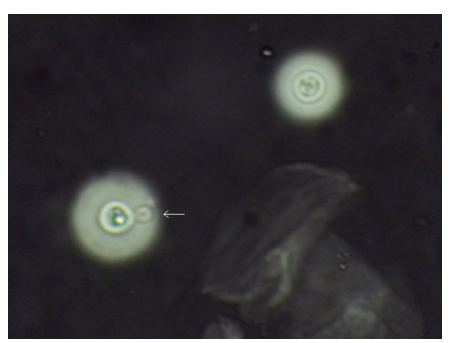

Figure 4: Sputum showing cryptococci on India Ink preperation. Budding yeast covered with capsule (white arrow).

She was found to be non-reactive for HIV 1 and 2. India ink staining of sputum revealed encapsulated yeast resembling cryptococci (Figure 4).

Sputum culture for fungus confirmed cryptococci. We reviewed the previous FNAC slides from lung space occupying lesion (SOL) which gave an impression of chronic granulomatous lesion showing large population of capsulated yeast cells morphologically resembling cryptococci (Figure 5), which was probably overlooked initially.

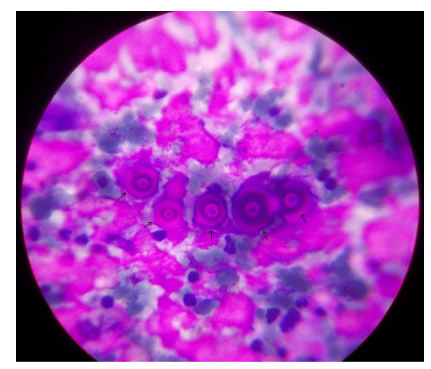

Figure 5: FNAC from left lung lesion showing cryptococci (black arrows).

Biopsy from the skin lesion also showed cryptococci with inflammatory cells (Figure 6).

As central nervous system (CNS) is one of the commonest sites involved in cryptococcosis, we did a cerebrospinal fluid (CSF) study although she never complained of any CNS symptom in her disease course.

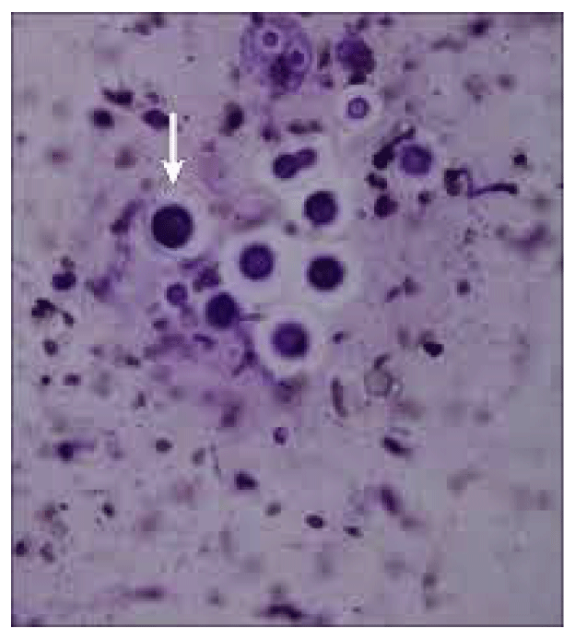

Figure 6: Skin biopsy showing cryptococci.

CSF was clear in appearance with a mildly increased opening pressure, cell count- 4 per cumm, all were lymphocytes, glucose-56 $\mathrm{mg} / \mathrm{dl}$, protein $40 \mathrm{mg} / \mathrm{dl}$. Both India ink preparation and cryptococcal antigen (CRAG) were positive in CSF with CRAG showing a titre of $1: 100$ (positive if $>1: 4$ ). MRI brain showed a few conglomerate lesions in right parietal region with ring enhancement and few such lesions in left perisylvian areas with cerebellar atrophy (Figure 7a). Intravenous Amphotericin-B (1 mg/kg body weight) and tablet flucytosine (100 $\mathrm{mg} / \mathrm{kg}$ body weight) in 4 divided doses were started and continued for 6 weeks (as per IDSA guideline) [3]. She started to improve symptomatically with healing of skin lesion (Figure 2b) cough subsided, hemoptysis stopped, appetite and general condition improved. Thereafter oral fluconazole $400 \mathrm{mg}$ for 8 weeks and then $200 \mathrm{mg}$ was continued for one year. After five months, there was 
significant regression of opacity on chest-X-ray (Figure 3b) and MRI brain also showed reduction in the size of the lesions (Figure $7 \mathrm{~b}$ ).

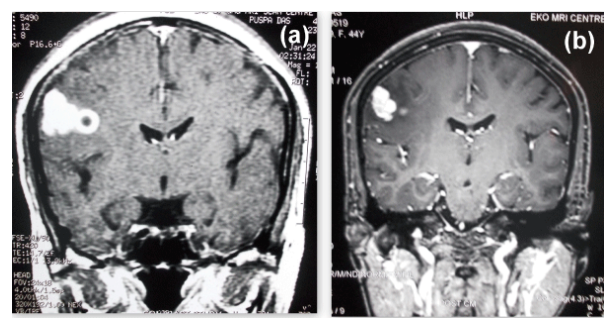

Figure 7: Showing MRI brain before (a) and after (b) treatment

She is still under our follow up and doing well without recurrence of symptoms.

\section{Sequence of Events}

Illness started with fever, dry cough, fatigue and weight loss. CT guided FNAC from left lung revealed granulomatous lesion without AFB. ATD started empirically.

$$
\downarrow \text { Two months later }
$$

Drug-induced hepatitis. ATD changed to hepatic-friendly regimen and continued for four months without improvement.

$$
\downarrow \text { One and half years after her initial symptoms }
$$

Patient developed carcinoma gall-bladder and was operated. A nodule seen on right arm which was gradually increasing in size in spite of treatment.

$$
\downarrow \text { Six months later }
$$

Biopsy done from the arm lesion which revealed Cryptococcus and she was referred to us. X-ray chest, CSF study, MRI brain done and treatment started.

$$
\downarrow \text { Five months later }
$$

Clinically improved, MRI brain and X-Ray chest revealed regression of the lesions.

\section{Discussion}

In immunocompetent persons, cryptococcosis is usually localised to lung as inhalation is the portal of entry. Cases of cryptococcal infections in immunocompetent hosts primarily include pulmonary and cutaneous lesions [4,5] and disseminated disease is rather uncommon. Invasive cryptococcosis occurs annually in (0.2-0.9) cases/ $100,000 \mathrm{HIV}$ negative persons and in $1700-6600$ cases per 100,000 persons with AIDS [6]. Incidence of Cryptococcal infection in cancer patients is only 0.5 cases per 100,000 [7]. It is evident from the above discussion that invasive cryptococcosis is a rare condition in both HIV negative and cancer patients as compared to AIDS. Our patient was negative for HIV infection and her clinical picture as narrated above does not raise any suspicion of immunosuppression. She developed gall bladder carcinoma about one and half year after initiation of her original illness; suggesting that the infection probably developed earlier than carcinoma of gall bladder.

As CNS is a potential site for Cryptococcal disease and it can be present alongwith pulmonary or skin lesions, it is prudent to rule out asymptomatic CNS infection in immunocompetent individuals. The case described here, had no CNS manifestation but both CSF and MRI imaging confirmed its presence. As a result lumbar puncture may be a useful tool to exclude concomitant CNS involvement [8].
On MRI, the commonest finding of cryptococcoma is punctate masses that demonstrate low signal intensity on T1-weighted images and high signal intensity on T2-weighted images, without surrounding edema [9]. The appearance of cryptococcus central nervous system infection in an immunocompetent patient may be different from that commonly encountered in the immunocompromised counterpart. Cryptococcosis should be considered in the differential diagnosis when multiple cystic lesions are noted in the basal ganglia, even in an immunocompetent patient. Marked ring enhancement of the basal ganglia lesions may be seen; this enhancement probably represents the patient's ability to mount an immune response, which would be uncommon in an immunocompromised patient. Marked surrounding edema may also be a feature of this infection [10]. Keeping in mind the atypical brain imaging appearance of our case, a granulomatous infection like tuberculoma would have been more appropriate possibility in Indian scenario. But considering her clinical background, we kept CNS cryptococcosis as primary possibility (patient did not consent to brain biopsy) and subsequently, positive response to treatment validated our diagnosis.

\section{Conclusion}

The case of disseminated cryptococcosis, we came across, is rare and unique in its presentation. Firstly, cryptococcosis may present as persistent lung and/or skin lesions in endemic areas simulating tuberculosis. Secondly, in all cases of pulmonary/cutaneous cryptococcosis, CNS involvement may occur even without any symptoms. Thirdly disseminated Cryptococcosis is possible even in the absence of known immunosuppressive conditions. Furthermore, cryptococcoma may also present as atypical appearance like ring and nodular enhancing lesions on neuroimaging.

\section{References}

1. Casadevall Arturo Cryptococcosis. In Fauci AS, Kasper DL, Longo DL, Braunwald dE, Hauser SL, et al. (2008) Harrison's Principles of Internal Medicine. 17th ed. McGraw-Hill Medical: New York: 1252-1259

2. King JW Cryptococcosis (2009) eMedicine. Available: http:// www.emedicine.com/med/topic 482. Accessed 30 October 2009.

3. Perfect JR, Dismukes WE, Dromer F, Goldman DL, Graybill JR, et al. (2010) Clinical practice guidelines for the management of cryptococcal disease: 2010 update by the infectious diseases society of America. Clin Infect Dis 50: 291-322

4. Capoor MR, Nair D, Deb M, Gupta B, Aggarwal P (2007) Clinical and mycological profile of cryptococcosis in a tertiary care hospital. Indian J Med Microbiol 25: 401-404.

5. Núñez M, Peacock JE Jr, Chin R Jr (2000) Pulmonary cryptococcosis in the immunocompetent host. Therapy with oral fluconazole: a report of four cases and a review of the literature. Chest 118: 527-534.

6. Hajjeh RA, Conn LA, Stephens DS (1999) Cryptococcosis: populationbased multistate active surveillance and risk factors in human immunodeficiency virus infected persons. J Infect Dis 179: 449-54.

7. Sebti A, Shah MK, Sepkowitz KA (2001) Invasive cryptococcosis in HIV negative patients at a cancer center [abstract]. In: Proceedings and abstracts of the 11th Annual Focus on Fungus, Washington DC.

8. Saag MS, Graybill RJ, Larsen RA, Pappas PG, Perfect JR, et al. (2000) Practice guidelines for the management of cryptococcal disease. Infectious Diseases Society of America. Clin Infect Dis 30: 710-718.

9. Farhood Saremi, James G Smirniotopoulos (2013) Imaging in CNS Cryptococcosis

10. Saigal G, Post MJ, Lolayekar S, Murtaza A (2005) Unusual presentation of central nervous system cryptococcal infection in an immunocompetent patient. AJNR Am J Neuroradiol 26: 2522-2526. 